\title{
As pesquisas abordando Ciências/Biologia em museus: Análise de uma década em revistas científicas
}

\author{
Research on Science/Biology in museums: An analysis of a decade in scientific journals \\ Las investigaciones que se acercan a las Ciencias/Biología en los museos: Análisis de una década en \\ las revistas científicas
}

\section{Resumo}

Os museus são importantes espaços que estimulam a reflexão, a criatividade e auxiliam no processo de construção do conhecimento e no ensino-aprendizagem de professores, alunos e comunidade. O objetivo do presente trabalho consistiu em realizar o estado da arte de artigos científicos dos anos de 2011 a 2020, em revistas de relevância, com a temática de ciências e biologia nos museus brasileiros, por meio de uma análise documental bibliográfica. As revistas analisadas possuem Qualis A1 e A2 da área de Ensino e Educação e foram escolhidas com base na Plataforma Sucupira da Coordenação de Aperfeiçoamento de Pessoal de Nível Superior - CAPES. Foram encontrados 46 artigos e a partir de suas análises, observou-se que a principal temática encontrada foi a de museus com as áreas de Ciências Naturais/geral, com maiores números de trabalhos no Museu de Astronomia e Ciências afins e Museu Espaço Ciência Viva, sendo a região Sudeste predominante com os estados do Rio de Janeiro e São Paulo. O principal público-alvo das pesquisas foram os funcionários dos museus e os professores do Ensino Fundamental e a análise qualitativa a abordagem mais utilizada. Os resultados encontrados revelam poucos trabalhos desenvolvidos sobre ciências/biologia em museus no Brasil, mas que os trabalhos existentes demonstram diversificação quanto aos espaços museológicos, ferramentas metodológicas para a obtenção dos dados e público-alvo, e reforçam a importância dos museus como locais de democratização da ciência.

Palavras-chave: Educação não formal; Espaços museológicos; Estado da arte. 


\begin{abstract}
Museums are important spaces that stimulate reflection, creativity and help in the process of knowledge construction and teaching-learning for teachers, students and the community. The objective of this work was to carry out a state of the art of scientific articles from 2011 to 2020, in relevant journals, with the theme of science and biology in Brazilian museums, through a bibliographic documentary analysis. The journals analyzed have qualis A1 and A2 in the area of Teaching and Education and they were chosen based on the Platform Sucupira of the Coordination for the Improvement of Higher Education Personnel - Capes. Forty-six articles were found and from their analysis, it was observed that the main theme found was museums with areas of natural/general sciences, with the largest number of works in the Museum of Astronomy and Related Sciences and Espaço Ciência Viva Museum, being the Southeast region predominant with the states of Rio de Janeiro and São Paulo. The main target audience of the research was museum staff and elementary school teachers, and qualitative analysis was the most used approach. The results found reveal few works developed about science/biology in museums in Brazil, but that the existing works demonstrate diversification regarding museum spaces, methodological tools for obtaining data and target audience, and reinforce the importance of museums as places of democratization of science.
\end{abstract}

Keywords: Non-formal education; Museum spaces; State of art.

\title{
Resumen
}

Los museos son espacios importantes que estimulan la reflexión, la creatividad y ayudan en el proceso de construcción del conocimiento y la enseñanza-aprendizaje de profesores, alumnos y comunidad. El objetivo del presente trabajo consiste en realizar el estado del arte de artículos científicos de los años 2011 a 2020, en revistas de relevancia, con la temática de ciencias y biología en los museos brasileños, mediante un análisis documental bibliográfico. Las revistas analizadas tienen Qualis A1 y A2 en el área de Enseñanza y Educación y fueron elegidas con base en la Plataforma Sucupira de la Coordinación para el Perfeccionamiento del Personal de Educación Superior - CAPES. Se encontraron 46 artículos y a partir de su análisis, se observó que el principal tema encontrado fue el de los museos con áreas de Ciencias Naturales/Generales, con mayor número de trabajos para los Museos de Astronomía y ciencias afines y en el Museo Espaço Ciência Viva, siendo la región Sudeste la predominante con los estados de Río de Janeiro y São Paulo. Los principales destinatarios de las investigaciones fueron los empleados de los museos y los profesores de primaria, y el análisis cualitativo fue el enfoque más utilizado. Los resultados muestran que se han desarrollado pocos estudios sobre ciencia/biología en los museos de Brasil, pero que los existentes demuestran una diversificación en cuanto a los espacios museísticos, las herramientas metodológicas para la obtención de datos y el público objeto de estudio; refuerzan la importancia de los museos como lugares para la democratización de la ciencia.

Palabras clave: Educación no formal; Espacios museísticos; Estado del arte.

\section{Introdução}

De acordo com Pscheidt e Lorenzetti (2020), os museus de ciências estão categorizados como espaços não formais e de disseminação de saberes, ou seja, são organizações de educação não formal que promovem vivências diversas e instigantes, proporcionando a aproximação das pesquisas que são desenvolvidas e o acesso aos elementos biológicos. Nesse sentido, os museus contribuem positivamente com a educação formal, a partir das visitações guiadas, onde é possível visitar as exposições e participar das práticas de educação. Ademais, isso também ocorre quando as famílias visitam os museus, havendo uma aproximação com o meio científico (Scalfi, Iszlaji \& Marandino, 2020).

O desenvolvimento de museus relacionados às áreas de Ciências e Tecnologia é bastante recente no Brasil, tendo se expandido nas últimas décadas e se adaptado às constantes mudanças do meio social (Valente, 2014). Segundo a mesma autora, essas adaptações devem priorizar o público participante do museu, as apresentações desenvolvidas, além das coleções que existem nesse ambiente. Os museus de ciências são espaços essenciais para a divulgação científica e para fortalecer as relações do desenvolvimento científico com o ensino.

Os centros de ciências e os museus desempenham um papel muito importante na formação dos professores, diante disso, muitos docentes ou as próprias instituições procuram esses espaços para a qualificação dos profissionais (Pugliese \& Marandino, 2015). De acordo com as autoras, por meio dessa capacitação, os educadores vivenciam diversas formas de entender e gerar ciência, assim, podem incentivar os estudantes a conhecerem mais os museus, contribuindo para que esses locais sejam vistos como essenciais no ensino de ciências. Para Scalfi, Iszlaji e Marandino (2020), visitas aos museus utilizando um ponto de vista da ciência, tecnologia, sociedade e meio ambiente, focando em questões sociais e científicas, podem contribuir para as mudanças 
no ensino, proporcionando inovações e reflexões para surpreender as aulas.

Outrossim, os museus de ciências devem ser espaços que contribuam para que os diversos públicos tenham acesso às atividades e exposições propostas. Dessa forma, segundo Bizerra et al (2012), os setores da educação desses espaços têm utilizado o tato e a audição nas atividades museais, promovendo inclusão do público cego ou com baixa visão, por meio da utilização de instrumentos e objetos em três dimensões, além de utilizarem áudios que servem como guia durante a visita.

Outro ponto que deve ser destacado é que as instituições de ensino, como universidades, devem identificar os museus de ciências como locais de conhecimento para os docentes da licenciatura por intermédio dos três pilares: pesquisa, ensino e extensão (Silva \& Leite, 2020). Ainda segundo as autoras, os museus atuam associando as suas práticas com os ensinamentos e as aprendizagens que ocorrem em sala de aula nas escolas, possibilitando ainda que os estudantes de graduação tenham contato com a divulgação científica, âmbito significativo no desenvolvimento dos docentes em ciências.

Considerando que são diversas as metodologias de diagnóstico e avaliação das atividades desenvolvidas nestes espaços e a qualidade em que/onde ocorrem, faz-se necessário desenvolver um trabalho sobre estado da arte sobre o assunto. Este dado, servirá não somente para o pesquisador, mas também para estudos futuros, uma vez que esta metodologia permite o conhecimento básico acerca do conhecimento produzido e problematizado, lacunas e as abordagens mais frequentemente utilizadas (Lazzarin, 2017; Fantin, Santos \& Martins, 2019).

Assim, o objetivo deste trabalho foi realizar o estado da arte de artigos científicos durante uma década (2011 a 2020) que abordam ensino de ciências e biologia nos museus nacionais, a partir da identificação de descritores durante a leitura dos artigos, visando compreender a principal perspectiva das publicações sobre museus.

\section{Metodologia}

Esta pesquisa resulta de uma análise documental de natureza bibliográfica, na qual a principal característica deste estudo é ser desenvolvida a partir de material já elaborado e constituído (Gil, 2008). O estudo configura um estado da arte, desenvolvido no intuito de mapear e discutir determinada temática do conhecimento, se utilizando da descrição produtiva, inventariante e sistematizada do assunto investigado (Ferreira, 2002; Romanowski \& Ens, 2006).

Esta pesquisa se fundamentou numa metodologia de natureza qualitativa que não se preocupa com representatividade numérica, mas com aspectos da realidade que não podem ser quantificados centrando-se na compreensão e explicação da dinâmica das relações sociais (Córdova, 2009). É o tipo de pesquisa que trabalha com o universo dos significados, dos motivos, das aspirações, das crenças, dos valores e das atitudes (Minayo, 2010).

O estudo foi realizado através do levantamento de artigos científicos publicados durante uma década (2011 a 2020) em 16 revistas de impacto. Como critérios de busca, foram inicialmente filtradas na plataforma Sucupira da Coordenação de Aperfeiçoamento de Pessoal de Nível Superior - CAPES, as revistas avaliadas nas Classificações de Periódicos Quadriênios 2013-2016, área de avaliação Ensino e Educação e com Qualis A1 e A2. Em seguida, foram selecionadas revistas com quantidade próxima e/ou superior a 30 artigos por ano e após a análise do cenário de 7.365, foram encontrados 44 artigos que abordam ciências e/ou biologia em museus do Brasil, listados no Quadro 1. 
Quadro 1. Revistas e artigos analisados abordando ciências e/ou biologia em museus do Brasil.

\begin{tabular}{|c|c|c|c|c|}
\hline Código & Revista & Edição & Título & Autores \\
\hline A1 & \multirow{6}{*}{$\begin{array}{l}\text { Ciência \& } \\
\text { Educação }\end{array}$} & 23(4), 2017 & $\begin{array}{l}\text { O discurso museológico e suas tipologias em um museu } \\
\text { de história natural }\end{array}$ & Pereira, B. de O. \& Do Valle, M. G. \\
\hline A2 & & 23(3), 2017 & $\begin{array}{l}\text { Educação ambiental e as dimensões cognitiva e afetiva } \\
\text { do trabalho com valores: produzindo sentidos }\end{array}$ & $\begin{array}{l}\text { Degasperi, T. C. \& Bonotto, D. M. } \\
\text { B. }\end{array}$ \\
\hline A3 & & 24(2), 2018 & $\begin{array}{l}\text { Ciência e teatro: um estudo sobre as artes cênicas como } \\
\text { estratégia de educação e divulgação da ciência em } \\
\text { museus }\end{array}$ & $\begin{array}{l}\text { Silva Almeida, C. da, Freire, M., } \\
\text { Bento, L., Jardim, G., Ramalho, M. } \\
\text { \& Dahmouche, M. }\end{array}$ \\
\hline A4 & & 25(2), 2019 & $\begin{array}{l}\text { O ensino de Sciencias Naturaes e os alunos surdos do } \\
\text { século XIX }\end{array}$ & Sofiato, C. G. \& Santana, R. S. \\
\hline A5 & & 25(4), 2019 & Diálogo com mediadores de Museus de Ciência & $\begin{array}{l}\text { Roldi, M. M. C., Silva, M. do. A. J. } \\
\text { \& Campos, C. R. P. }\end{array}$ \\
\hline A6 & & $\begin{array}{l}26(\mathrm{e} 20050) \\
2020\end{array}$ & $\begin{array}{l}\text { O Público Potencial Escolar do Museu da Vida: um } \\
\text { estudo exploratório em escolas da zona norte da cidade } \\
\text { do Rio de Janeiro }\end{array}$ & Cabral, E. da C. \& Guimarães, V. F. \\
\hline A7 & $\begin{array}{l}\text { Educação e } \\
\text { Pesquisa }\end{array}$ & $\begin{array}{l}\text { 44(e170831) } \\
2018\end{array}$ & $\begin{array}{l}\text { Alfabetização científica, criança e espaços de educação } \\
\text { não formal: diálogos possíveis }\end{array}$ & $\begin{array}{l}\text { Marques, A. C. T. L. \& Marandino, } \\
\text { M. }\end{array}$ \\
\hline A8 & $\begin{array}{c}\text { Educação em } \\
\text { Revista (UFMG) }\end{array}$ & $\begin{array}{l}36(\mathrm{e} 234507) \\
2002\end{array}$ & $\begin{array}{l}\text { A educação inclusiva em espaços não formais: Uma } \\
\text { análise dos museus de ciências brasileiros }\end{array}$ & Schuindt, C. C. \& Silveira, C. \\
\hline A9 & \multirow{7}{*}{$\begin{array}{l}\text { Ensaio: Pesquisa } \\
\text { em Educação em } \\
\text { Ciências }\end{array}$} & 13(3), 2011 & $\begin{array}{l}\text { Prática de ensino de Ciências: O museu como espaço } \\
\text { formativo }\end{array}$ & Ovigli, D. F. B. \\
\hline A10 & & 14(1), 2012 & $\begin{array}{l}\text { Modelos de educação em Ciências em museus: Análise } \\
\text { da visita orientada }\end{array}$ & "Marandino, M. \& Ianelli, I. T. \\
\hline A11 & & 18(1), 2016 & $\begin{array}{l}\text { Formação de mediadores em museus de Ciência: } \\
\text { Saberes e práticas }\end{array}$ & Gomes, I. \& Cazelli, S. \\
\hline A12 & & $\begin{array}{l}19(\mathrm{e} 2470) \\
2017\end{array}$ & $\begin{array}{l}\text { Formação continuada de professores dos anos iniciais } \\
\text { da educação básica: impacto do programa formativo de } \\
\text { um museu de ciência a partir do viés crítico-reflexivo }\end{array}$ & $\begin{array}{l}\text { Pereira, G. R., Paula, L. M., Paula, } \\
\text { L. M. \& Silva, R. C. }\end{array}$ \\
\hline A13 & & $\begin{array}{l}20(\mathrm{e} 8684) \\
\quad 2018\end{array}$ & $\begin{array}{l}\text { A compreensão da biodiversidade por meio dioramas } \\
\text { de museus de Zoologia: Um estudo com o público } \\
\text { adulto no Brasil e na Dinamarca }\end{array}$ & Marandino, M. \& Laurini, C. \\
\hline A14 & & $\begin{array}{l}21(\mathrm{e} 10524) \\
2019\end{array}$ & $\begin{array}{l}\text { A Experiência de adolescentes ao visitar um museu de } \\
\text { ciência: Um estudo no Museu da Vida }\end{array}$ & $\begin{array}{l}\text { Massarani, L., Reznik, G., Rocha, J. } \\
\text { N., Falla, S., Rowe, S., Martins, A. } \\
\text { D. \& Amorim, L. H. }\end{array}$ \\
\hline A15 & & $\begin{array}{l}22(\mathrm{e} 13514) \\
2020\end{array}$ & $\begin{array}{l}\mathrm{O} \text { museu ciência e vida investiga seu público: } \\
\text { Professores }\end{array}$ & $\begin{array}{l}\text { Dahmouche, M. S., Pires, A. M. G. } \\
\text { \& Cazelli, S. }\end{array}$ \\
\hline A16 & \multirow{2}{*}{$\begin{array}{l}\text { Investigações em } \\
\quad \text { Ensino de } \\
\text { Ciências (IENCI) }\end{array}$} & 19(2), 2014 & $\begin{array}{l}\text { Visitas guiadas ao museu da } \\
\text { promovendo a cultura científica e } \\
\text { estudantes do Ensino Médio }\end{array}$ & $\begin{array}{l}\text { Oliveira, G. C. G., Turci, C. C., } \\
\text { Silva, F. S. S., Oliveira, L. S. C. \& } \\
\text { Abrey, S. D. }\end{array}$ \\
\hline A17 & & 20(3), 2015 & $\begin{array}{l}\text { O método da lembrança estimulada como uma } \\
\text { ferramenta de investigação sobre a visita escolar no } \\
\text { Museu de Biodiversidade do Cerrado }\end{array}$ & Oliveira, L. M. \& Carvalho, D. F. \\
\hline A18 & \multirow{4}{*}{$\begin{array}{l}\text { Investigações em } \\
\quad \text { Ensino de } \\
\text { Ciências (IENCI) }\end{array}$} & 20(1), 2015 & $\begin{array}{l}\text { A aprendizagem num museu de ciência e o papel do } \\
\text { monitor. }\end{array}$ & $\begin{array}{l}\text { Carvalho, T. F. G. C. \& Pacca, J. L. } \\
\text { A. }\end{array}$ \\
\hline A19 & & 22(1), 2017 & $\begin{array}{l}\text { Contribuições de um museu de ciências para a } \\
\text { formação docente em física }\end{array}$ & Tempesta, A. M. \& Gomes, C. L. \\
\hline $\mathrm{A} 20$ & & 24(3), 2019 & $\begin{array}{l}\text { Os espaços não formais amazônicos como } \\
\text { potencializadores de aprendizagem para o ensino de } \\
\text { ciências: uma perspectiva a partir da teoria } \\
\text { fundamentada }\end{array}$ & Oliveira, A. R. H. R. \& Silva, C. C. \\
\hline $\mathrm{A} 21$ & & $25(3), 2020$ & $\begin{array}{l}\text { Interações do público escolar em dois diferentes tipos } \\
\text { de museus de ciências }\end{array}$ & Masseto, E. \& Rosa, R. T. D. \\
\hline A22 & $\begin{array}{c}\text { Revista } \\
\text { Educação } \\
\text { Especial }\end{array}$ & 25(42), 2012 & $\begin{array}{l}\text { Conversas de aprendizagem em museus de ciências: } \\
\text { como os deficientes visuais interpretam os materiais } \\
\text { educativos do museu de microbiologia? }\end{array}$ & $\begin{array}{l}\text { Bizerra, A. F., Cizauskas, J. B. V., } \\
\text { Inglez, G. C. \& Franco, M. T. }\end{array}$ \\
\hline A23 & \multirow{2}{*}{$\begin{array}{l}\text { Atos de Pesquisa } \\
\text { em Educação } \\
\text { (FURB) }\end{array}$} & $9(3), 2014$ & $\begin{array}{l}\text { O museu como espaço de constituição da formação } \\
\text { docente em ciências e biologia }\end{array}$ & $\begin{array}{l}\text { Sousa, C. E. B. \& Carvalho Neta, R. } \\
\text { N. F. }\end{array}$ \\
\hline A24 & & 13(1), 2018 & $\begin{array}{l}\text { As crianças e o guia dos museus brasileiros como } \\
\text { objetos de estudo }\end{array}$ & Carvalho, C. \\
\hline
\end{tabular}




\begin{tabular}{|c|c|c|c|c|}
\hline A 25 & & 15(3), 2020 & $\begin{array}{l}\text { Formação de professores em museus de ciências: } \\
\text { Construindo o estado da questão }\end{array}$ & Silva, M. C. B. \& Leite, R. C. M. \\
\hline A26 & \multirow{5}{*}{$\begin{array}{l}\text { Esino em Re- } \\
\quad \text { Vista }\end{array}$} & 20(1), 2013 & $\begin{array}{l}\text { Representações de educação não formal e utilização do } \\
\text { espaço museal por professoras do ensino fundamental }\end{array}$ & $\begin{array}{lllll}\text { Jacobucci, } & \text { D., } & \text { Ferreira, } & \text { F. } & \& \\
\text { Santana, F. } & & & & \end{array}$ \\
\hline A27 & & 20(1), 2013 & $\begin{array}{l}\text { Formação de mediadores para museus em situações } \\
\text { educacionais ampliadas: saberes da mediação e } \\
\text { desenvolvimento profissional }\end{array}$ & Queiroz, G. \\
\hline A28 & & 20(1), 2013 & $\begin{array}{l}\text { A relação museu e escola: um duplo olhar sobre a ação } \\
\text { educativa em seis museus de minas gerais }\end{array}$ & Nascimento, $\mathrm{S}$. \\
\hline A29 & & 20(1), 2013 & O Programa Educativo do Museu de Astronomia & $\begin{array}{l}\text { Silva, D., Coimbra, C., Cazelli, S. \& } \\
\text { Valente, M. }\end{array}$ \\
\hline $\mathrm{A} 30$ & & 20(1), 2013 & $\begin{array}{l}\text { Museu de ciências universitário: sobre espaços de } \\
\text { divulgação, educação e produção científica }\end{array}$ & Rossi, A. \\
\hline $\mathrm{A} 31$ & \multirow{3}{*}{ Revista Práxis } & $7(14), 2015$ & $\begin{array}{l}\text { Educação Científica e Ambiental através de diálogos e } \\
\text { práticas no Museu de História Natural da Universidade } \\
\text { Federal de Lavras }\end{array}$ & $\begin{array}{l}\text { Melo, J. S. A., Ferreira, L. N. \& } \\
\text { Nascimento Júnior, A. F. }\end{array}$ \\
\hline A32 & & 11(21), 2019 & $\begin{array}{l}\text { Museu do Amanhã: uma investigação tátil com o olhar } \\
\text { às cegas de quem vê }\end{array}$ & $\begin{array}{l}\text { Júnior, E. S. S., Braga, A. N., Bahia, } \\
\text { S. R., Pinto, S. C. S. \& Braz, R. M. } \\
\text { M. }\end{array}$ \\
\hline A33 & & 12(1), 2020 & $\begin{array}{l}\text { Museus de ciências em tempos de pandemia: uma } \\
\text { análise no Instagram do museu da vida }\end{array}$ & $\begin{array}{l}\text { Freitas, T. P. R., Silveira, J. B. A., } \\
\text { Costa, P. P. M., Miceli, B. S. \& } \\
\text { Rocha, M. B. }\end{array}$ \\
\hline A34 & \multirow{3}{*}{$\begin{array}{c}\text { Revista } \\
\text { Brasileira de } \\
\text { Ensino de } \\
\text { Ciência e } \\
\text { Tecnologia } \\
\text { (RBECT) }\end{array}$} & 6(2), 2013 & $\begin{array}{l}\text { Programa "ciência vai à escola" - museu de ciências } \\
\text { naturais da UFPR: construindo uma visão de ciência na } \\
\text { educação básica }\end{array}$ & $\begin{array}{l}\text { Pauliv, V., Carvalho, L., Felippe, C., } \\
\text { Bobato, R. \& Sedor, F. }\end{array}$ \\
\hline A35 & & 12(2), 2019 & $\begin{array}{l}\text { A escola vai ao museu: ações e percepções dos } \\
\text { professores nesse percurso }\end{array}$ & $\begin{array}{l}\text { Oliveira, G., Guimarães, P. \& } \\
\text { Monteiro, P. }\end{array}$ \\
\hline A36 & & 13(1), 2020 & $\begin{array}{l}\text { As contribuições das ilhas interdisciplinares de } \\
\text { racionalidade na relação museu-escola }\end{array}$ & Carneiro, G. \& Cavassan, O. \\
\hline A 37 & \multirow{5}{*}{$\begin{array}{c}\text { Revista } \\
\text { Brasileira de } \\
\text { Pesquisa em } \\
\text { Educação em } \\
\text { Ciências }\end{array}$} & 1191), 2011 & $\begin{array}{l}\text { Ensino e aprendizagem de Biologia em trilhas } \\
\text { interpretativas: o modelo contextual do aprendizado } \\
\text { como referencial }\end{array}$ & $\begin{array}{l}\text { De Araujo, E. S. N. N., Soman, J. } \\
\text { M., Caluzi, J. C. \& Caldeira, A. M. } \\
\text { de. A. }\end{array}$ \\
\hline A38 & & 12(3), 2012 & $\begin{array}{l}\text { A educação não formal no Brasil: o que apresentam os } \\
\text { periódicos em três décadas de publicação (1979-2008) }\end{array}$ & $\begin{array}{l}\text { Passos, M. M., Arruda, S. de M. \& } \\
\text { Alves, D. R. S. }\end{array}$ \\
\hline A39 & & 17(2), 2017 & $\begin{array}{l}\text { Estudo de públicos sobre aprendizagens genéricas } \\
\text { promovidas por uma exposição de nanotecnologia em } \\
\text { Pernambuco }\end{array}$ & De Morais, C. S.; \& Ferreira, H. S. \\
\hline A40 & & 18(1), 2018 & $\begin{array}{l}\text { Learning about Microorganisms in Childhood: Fourto } \\
\text { Six-Year-Old Children's Voice in Kindergartens and } \\
\text { Museums }\end{array}$ & $\begin{array}{l}\text { Dominguez, C. R. C., Leporo, N., De } \\
\text { Franco, M. T., Inglez, G. C., } \\
\text { Gonçalves, V. M. \& Bizerra, A. F. }\end{array}$ \\
\hline A41 & & 18(3), 2018 & $\begin{array}{l}\text { Ação Mediada e Ensino por Investigação: Um Estudo } \\
\text { Junto a Alunos do Ensino Médio em um Museu de } \\
\text { Ciências }\end{array}$ & $\begin{array}{l}\text { Roldi, M. M. C., Silva, M. do A. J. } \\
\text { \& Trazzi, P. S. S. }\end{array}$ \\
\hline A42 & Revista Exitus & $\begin{array}{l}10(\mathrm{e} 020096) \\
2020\end{array}$ & $\begin{array}{l}\text { Exposição museológica "Transformações: a Amazônia } \\
\text { e o Antropoceno." Objetos de conhecimentos e sua } \\
\text { relação com o ensino de ciências. }\end{array}$ & $\begin{array}{l}\text { Freitas, R. F., Oliveira, E. M. \& } \\
\text { Freitas, N. M. S. }\end{array}$ \\
\hline A43 & \multirow{2}{*}{$\begin{array}{l}\text { Ensino, Saúde e } \\
\text { Ambiente }\end{array}$} & 7(3), 2014 & $\begin{array}{l}\text { Professores e o museu de biodiversidade do cerrado: } \\
\text { vínculos através da construção coletiva de recursos } \\
\text { lúdicos }\end{array}$ & Santos, L. V. \& Carvalho, D. F. \\
\hline A44 & & $9(3), 2016$ & $\begin{array}{l}\text { O entorno que não vai: um estudo de caso do não- } \\
\text { público de um museu de ciências no rio de janeiro }\end{array}$ & $\begin{array}{l}\text { Paula, L. M., Pereira, G. R., Paula, } \\
\text { L. M. \& Silva, R. C. }\end{array}$ \\
\hline
\end{tabular}

Fonte: Autores (2021).

Os dados provenientes dos artigos foram examinados visando identificar informações referentes à temática, local de realização (incluindo museu, cidade e estado), público-alvo e abordagens e ferramentas metodológicas. Em seguida, estas informações foram organizadas em tabelas e gráficos utilizando o programa Microsoft® Excel 2013. 


\section{Resultados e Discussão}

Em relação à temática dos artigos analisados, a primeira área com maior abordagem foi a de Ciências Naturais, com presença de 59,09\%, seguida pela Biodiversidade, apresentando 11,36\% dos estudos, e as terceiras maiores áreas foram de Educação Ambiental e de Divulgação Científica indicando 9,09\% dos artigos em ambas (Figura 1).

É possível observar que durante as décadas de 1950 e 1960 houve a institucionalização das ciências e em seguida criados museus de ciência e tecnologia, visando reunir diferentes âmbitos da comunidade científica, cultural, política e sociedade (Cancela \& Carvalho, 2017), assim, o surgimento destes museus mais gerais pode justificar a maior percentagem identificada neste estudo uma vez que estes espaços são mais comuns que os mais específicos.

Figura 1. Temática dos artigos analisados que abordam ciências e/ou biologia em museus do Brasil.

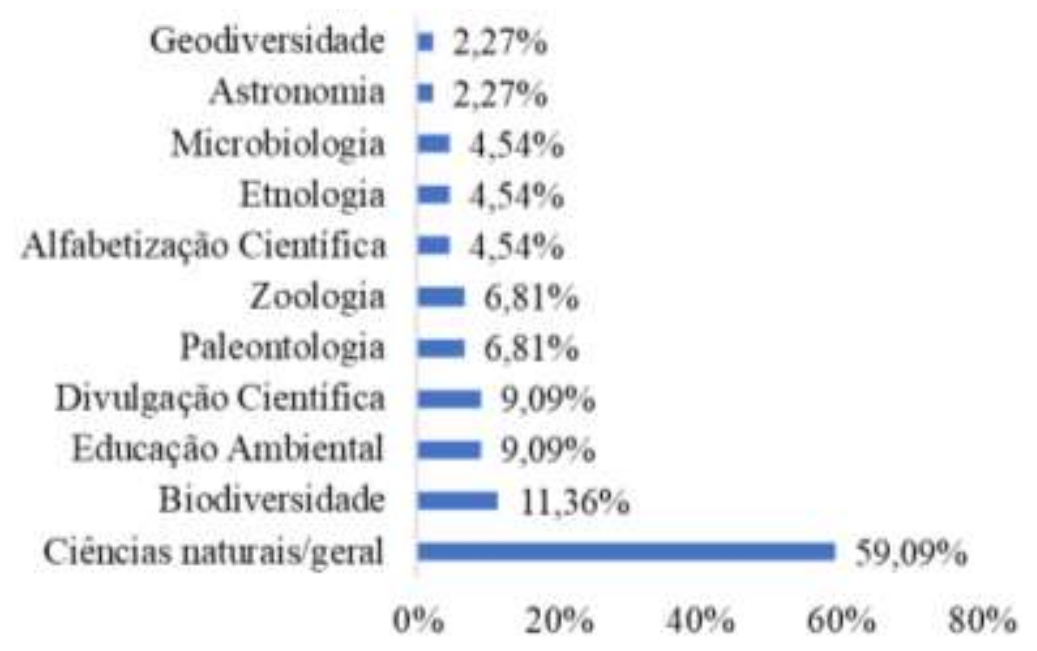

Fonte: Autores (2021).

Desse modo, segundo Ovigli (2015), 80\% dos trabalhos realizados durante os anos de 2000 a 2010 foram acerca de educação em museus de ciências. Acredita-se que isso ocorreu devido à formação da área 46 (Ensino) pela Capes e à grande proposta de cursos com particularidades relacionadas aos museus e à educação. Para ele, a elaboração universitária sobre esse tema é amplamente fortalecida com o surgimento de áreas e de cursos, além do estabelecimento de programas já presentes no Brasil, que aumentam as oportunidades, e do desenvolvimento de mais museus de ciências nas regiões de todo o País.

A biodiversidade, segunda temática mais encontrada dentre os artigos, sempre esteve presente nas abordagens dos museus a partir dos Gabinetes de Curiosidades que iniciaram no século XVI. Ademais, é um tema que está inserido constantemente nas atividades hodiernas dos centros de cultura científica e dos museus de História Natural, por meio das exposições onde o público se aproxima dos assuntos relacionados à biodiversidade, analisando, elaborando e restabelecendo as visões sobre o assunto (Marandino et al., 2012), havendo bastante troca de conhecimentos e aprendizagens.

De acordo com Meyer e Meyer (2014), a área de educação ambiental é abordada nos museus de ciências, porém apresenta adversidades para ser colocada em prática de forma crítica, impossibilitando reflexões mais profundas do público. Para eles, isso ocorre devido à escassez de divulgação de informações nos museus de ciências sobre os complexos fatores responsáveis pela crise ambiental. Dessa forma, muitas vezes esses espaços ficam presos às informações técnicas, superficiais e simplistas, que não contribuem de fato para a formação de diálogos sobre a importância da educação ambiental. No entanto, mesmo com essas problemáticas, é muito importante que os museus busquem retratar a educação ambiental de maneira eficaz, havendo um compartilhamento de conteúdos críticos e verdadeiros, e que mantenham parceria entre os outros museus, para que haja uma fortificação dos laços da educação ambiental. 
A divulgação científica é uma área que tem crescido bastante dentro dos museus de ciências, nos últimos anos, devido à aproximação e expansão desses espaços junto das universidades, centros de ciências e instituições que trabalham com a ciência. Desse modo, a divulgação da ciência por meio de exposições, atividades com linguagem e comunicação acessível e transposição didática, em espaços como os museus, tornou possível o estabelecimento de uma relação mais próxima da sociedade com a ciência, já que durante muito tempo essas esferas caminhavam totalmente separadas (Rossi, 2013).

Os espaços museais estão intimamente relacionados à própria divulgação científica, a partir das informações encontradas nos museus, o público aprende e realiza ações. Além dos trabalhos executados pelos pesquisadores nesses locais, eles são muito importantes para a preservação do patrimônio histórico e da biodiversidade. Assim, é necessário divulgar as informações de forma simples, para que elas sejam compreendidas, e não apenas um meio de entreter os visitantes, a verdadeira divulgação científica é realizada quando há troca de conhecimentos quando o complexo é abordado de forma fácil, contribuindo para que as informações possam ser divulgadas e espalhadas pela população (Falaschi, Capellari \& Oliveira, 2011). É imprescindível que os museus sejam acessíveis com o objetivo de relacionar o conhecimento científico com as atividades do diaa-dia de cada visitante, dessa forma, as pesquisas científicas dos museus poderão ser compreendidas pela sociedade, já que a linguagem utilizada será simples, contribuindo para que o público possa se aprofundar e utilizar os museus como guias para resolver incertezas do cotidiano (Coelho et al, 2019).

Ao ser analisado o local de realização dos estudos, foram identificadas diversas instituições com atividades museológicas, na qual as com maiores destaques e quantidades de trabalhos estão situadas no estado do Rio de Janeiro, sendo estes: Museu de Astronomia e Ciências Afins - MAST e Museu Espaço Ciência Viva - ECV (Tabela 1).

Tabela 1. Local de realização dos artigos analisados que abordam ciências e/ou biologia em museus do Brasil.

\begin{tabular}{|c|c|c|}
\hline Estado & Museu/Centro de Ciências & Quantidade \\
\hline \multirow[t]{2}{*}{ Amazonas } & Museu Amazônico da UFAM & 1 \\
\hline & O Bosque da Ciência do INPA & 1 \\
\hline Ceará & Museu Seara da Ciência da UFC & 1 \\
\hline Espírito Santo & Museu de Biologia Professor Mello Leitão do INMA & 2 \\
\hline \multirow[t]{2}{*}{ Maranhão } & Centro de Pesquisa de História Natural e Arqueologia do Maranhão & 2 \\
\hline & Laboratório de Divulgação Científica Ilha da Ciência & 1 \\
\hline Mato Grosso do Sul & Casa da Ciência e Cultura de Campo Grande & 1 \\
\hline \multirow[t]{3}{*}{ Minas Gerais } & Museu de Biodiversidade do Cerrado da UFU & 3 \\
\hline & Museu de História Natural da UFLA & 1 \\
\hline & Outros museus não especificados & 1 \\
\hline \multirow[t]{2}{*}{ Pará } & Centro de Ciências e Planetário do Pará & 1 \\
\hline & Museu Paraense Emílio Goeldi & 1 \\
\hline \multirow[t]{2}{*}{ Paraná } & Museu Dinâmico Interdisciplinar da UEM & 3 \\
\hline & Museu de Ciências Naturais da UFPR & 1 \\
\hline Pernambuco & Espaço Ciência - Museu Interativo de Ciência de Pernambuco & 1 \\
\hline Piauí & Museu Itinerante de Química do IFPI & 1 \\
\hline \multirow[t]{10}{*}{ Rio de Janeiro } & Museu de Astronomia e Ciências Afins & 5 \\
\hline & Museu Espaço Ciência Viva & 5 \\
\hline & Museu da Vida da FIOCRUZ & 4 \\
\hline & Espaço Ciência InterAtiva do IFRJ & 3 \\
\hline & Museu Ciência e Vida - Fundação CECIERJ & 3 \\
\hline & Casa da Ciência da UFRJ & 2 \\
\hline & Espaço COPPE Miguel de Simoni da UFRJ & 1 \\
\hline & Fundação Planetário da Cidade do Rio de Janeiro & 1 \\
\hline & Museu do Amanhã & 1 \\
\hline & Museu do Meio Ambiente & 1 \\
\hline
\end{tabular}




\begin{tabular}{lll} 
& Museu do Horto & 1 \\
Museu do Universo da Cidade do Rio de Janeiro & 1 \\
\hline São Paulo & Museu de Ciências e Tecnologia da PUCRS & 4 \\
& Museu de Microbiologia do Instituto Butantan & 2 \\
& Museu de Zoologia da USP & 2 \\
& Espaço Interativo do Centro de Biotecnologia Molecular Estrutural & 1 \\
& Estação Ciências da PRCEU/USP & 1 \\
Museu Biológico do Instituto Butantan & 1 \\
& Museu da Floresta Estadual Edmundo Navarro de Andrade & 1 \\
& Museu do Café de Piratininga & 1 \\
Miferentes estados* & Museu Exploratório de Ciências da UNICAMP forma geral & 4 \\
\hline
\end{tabular}

Legenda: UFAM: Universidade Federal do Amazonas; INPA: Instituto Nacional de Pesquisa da Amazônia; UFC: Universidade Federal do Ceará; INMA: Instituto Nacional da Mata Atlântica; UFU: Universidade Federal de Uberlândia; UFLA: Universidade Federal de Lavras; UEM: Universidade Estadual de Maringá; UFPR: Universidade Federal do Paraná; IFPI: Instituto Federal de Educação, Ciência e Tecnologia do Piauí; FIOCRUZ: Fundação Oswaldo Cruz; IFRJ: Instituto Federal de Educação, Ciência e Tecnologia do Rio de Janeiro; CECIERJ: Centro de Ciências e Educação Superior a Distância do Estado do Rio de Janeiro; COPPE: Instituto Alberto Luiz Coimbra de Pós-Graduação e Pesquisa de Engenharia; UFRJ: Universidade Federal do Rio de Janeiro; PUCRS: Pontifícia Universidade Católica do Rio Grande do Sul; USP: Universidade de São Paulo; PRCEU: Pró-Reitoria de Cultura e Extensão Universitária; UNICAMP: Universidade Estadual de Campinas.

Fonte: Autores (2021). * Estados não especificados no trabalho.

O MAST (2021) é um espaço importante de acesso ao conhecimento científico e tecnológico para a sociedade que, por meio dos seus estudos e acervo, consegue preservar e compartilhar importantes momentos e atividades da ciência do estado do Rio de Janeiro e do Brasil com seus visitantes. Além de exposições e divulgação científica, o museu atrai muita atenção e visitação pois abrange diferentes áreas do conhecimento, como química, física, biologia e matemática, desenvolve pesquisas na área da ciência e educação, capacitação, oficinas e outros eventos.

O ECV apresenta também um espaço que se destaca quanto a democratização do conhecimento científico no Rio de Janeiro, responsável por divulgar ciência e proporcionar a participação e desenvolvimento de projetos científicos e de extensão com a sociedade, comunidade científica e acadêmica e pesquisadores de outras instituições (Costa, 2019).

Diante disso, é visível a diversificação destes espaços em grande parte do país, e a sua importância como ambientes não formais, que mesmo não sendo um museu institucionalizado, possibilita a visitantes, como estudantes por exemplo, desenvolver pensamentos e vivências dentro do conhecimento científico e perceber suas relações com o meio ambiente (Queiroz et al., 2011), como foi visto no presente estudo.

Embora na pesquisa não tenham sido encontrados trabalhos sobre os Ecomuseus, esses espaços são muito importantes para a ciência e tiveram sua criação a partir das dificuldades que a ecologia estava enfrentando, já que era uma área nova e que tinha como objetivo abordar o uso sustentável dos recursos presentes no planeta sem interferir nas gerações que ainda viriam, além disso, na época retratava-se muito sobre a função da ciência e da educação para o crescimento futuro. Assim, os Ecomuseus surgiram em um momento em que se discutia acerca das atribuições dos espaços museais para a sociedade e sobre como a população é estimulada pelas suas memórias, assim, eles refletem a transdisciplinaridade, a comunidade e o meio ambiente (Leite, 2016).

Sobre os estados e as regiões onde os museus analisados nos artigos estão localizados, houve um predomínio da região Sudeste $(79,54 \%)$, sendo 34,09\% dos trabalhos desenvolvidos em museus no estado do Rio de Janeiro, seguida por São Paulo com 29,54\%. O Nordeste é a segunda região que mais apareceu nas pesquisas (29,54\%), dentre seus estados destaca-se Pernambuco com 18,18\% dos trabalhos, considerado o estado com mais pesquisas na Região e o terceiro do país (Figura 2). 
Figura 2. Cidade/Estado de realização dos artigos analisados que abordam ciências e/ou biologia em museus do Brasil.

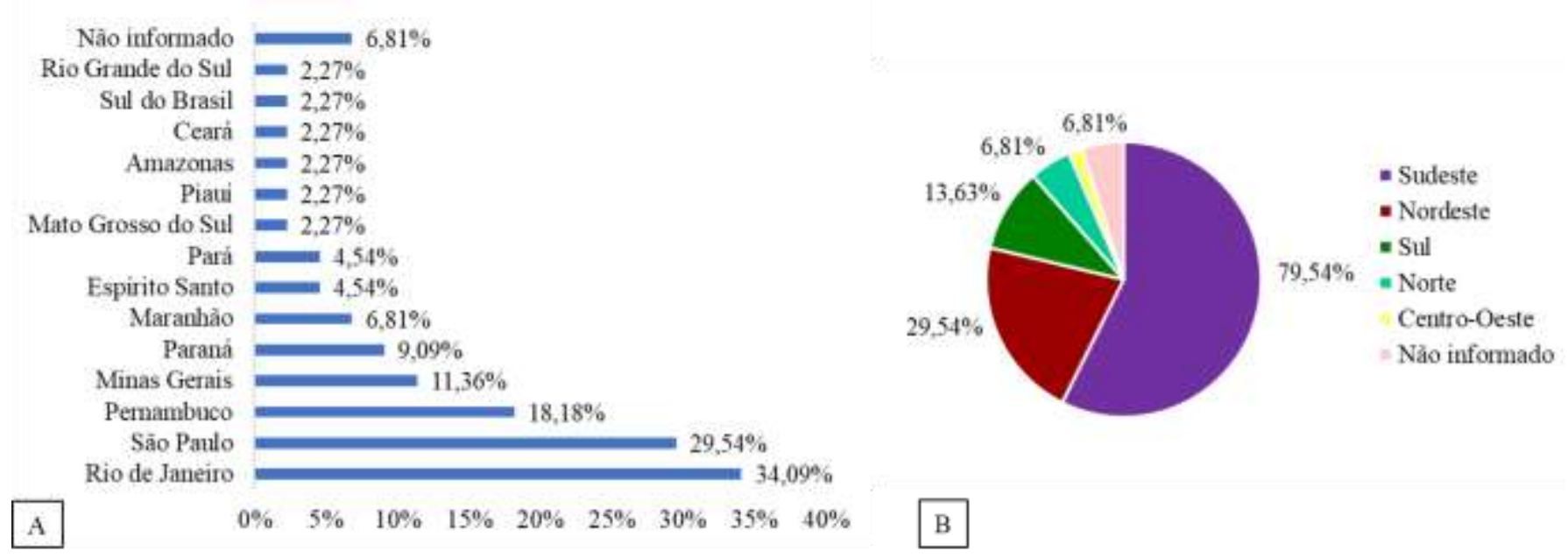

Fonte: Autores (2021).

Conforme os resultados apresentados no trabalho de Silva e Leite (2020) que corroboram com os desta pesquisa, notouse uma hegemonia de estudos acerca da educação nos museus de ciências na região Sudeste e nos estados de Rio de Janeiro e de São Paulo, nos quais apresentam grande parte dos museus de ciências do país. Segundo Steola e Kasseboehme (2018), a região Sudeste apresenta maior número de universidades o que possibilita o estabelecimento de parcerias com esses espaços não formais e estimula uma maior criação dos mesmos, ademais o Sudeste do Brasil ainda se destaca pelo seu alto desenvolvimento científico e tecnológico, o que explica uma maior concentração dos espaços museus nesta região.

Mesmo com o movimento da popularização da ciência ao redor das regiões do Brasil, através da inserção de políticas públicas pelos governos federal e estadual, ainda é notório a disparidade que também é refletida na distribuição dos museus, centros de ciências e outros espaços informais, pois estão localizados principalmente nas regiões Sudeste e Sul (ABMC, Casa das Ciências, Fiocruz \& Museu da Vida, 2015), havendo menos espaços de divulgação de ciências nas outras regiões do país, como o Nordeste, embora essa região tenha aparecido como a segunda com mais trabalhos sobre os museus de ciências e de biologia. Nesse sentido, existem outros museus, que não foram explorados nesta pesquisa, mas que devem ser estudados e avaliados, principalmente em relação aos vínculos que eles têm com as escolas pois quando essa parceria é consolidada, os alunos e professores tendem a ser muito beneficiados, já que a educação não formal nesses espaços contribui muito para enriquecer as experiências e os conhecimentos dos educandos e dos educadores.

No tocante ao público-alvo, representado pela Tabela 2, houve um predomínio dos funcionários do museu e dos professores do Ensino Fundamental, indicando que cerca de 31,81\% dos artigos abordaram esses públicos nos trabalhos. Em terceiro lugar, com $25 \%$ dos estudos, aparecem os alunos do Ensino Fundamental como sujeitos das pesquisas.

Nessa conjuntura, os funcionários dos museus desempenham um papel essencial na explicação do espaço para o público. Para Gomes e Cazelli (2016), os mediadores devem dominar as temáticas das exposições para instruir os visitantes, ou seja, é necessário que tenham um conhecimento do conteúdo para que aquele assunto abordado na exposição não seja apenas transmitido para o público, mas que haja uma interação entre mediador e visitante, por meio de um bom diálogo. Além disso, existem duas habilidades essenciais para a mediação nos museus, elas são a apresentação do contexto dos conteúdos apresentados e a comunicação eficaz nas atividades museais (Ovigli, 2011), a partir delas, a interação entre o público e os funcionários dos museus será bastante proveitosa. 
Tabela 2. Informações sobre o público-alvo investigado nos artigos analisados que abordam ciências e/ou biologia em museus do Brasil.

\begin{tabular}{l|cc|c}
\hline Investigados & Quantidade & $\%$ & $\begin{array}{c}\text { N. amostral dos participantes } \\
\text { investigados nos artigos }\end{array}$ \\
\hline Funcionários do museu * & 14 & $31,81 \%$ & 91 \\
Professores do Ensino Fundamental & 14 & $31,81 \%$ & 239 \\
Professores do Ensino Médio & 8 & $18,18 \%$ & 226 \\
Professores do Ensino Superior & 7 & $15,90 \%$ & 78 \\
Alunos do Ensino Fundamental & 11 & $25 \%$ & 195 \\
Alunos do Ensino Médio & 7 & $15,90 \%$ & 101 \\
Alunos do Ensino Superior & 6 & $13,63 \%$ & 26 \\
Comunidade escolar stricto sensu $* *$ & 7 & $15,90 \%$ & 19 \\
Comunidade escolar lato sensu $* * *$ & 6 & $13,63 \%$ & 217 \\
Pessoas com deficiência **** & 3 & $6,81 \%$ & - \\
Não informado & 2 & $4,54 \%$ & 17 \\
\hline
\end{tabular}

Legenda: * Mediadores, pesquisadores, monitores e curadores do museu; ** Diretores, coordenadores, bibliotecária; *** Pais dos alunos, comunidade em geral; **** Portadores de deficiência visual e/ou auditiva; - quantidade não informada.

Fonte: Autores (2021).

Os museus sendo espaços de construção de conhecimento que estimulam o debate e o diálogo, são essenciais no processo de formação do professor, seja ele ainda estudante de graduação ou já atuante no ambiente escolar. A partir dos anos 1980, as equipes dos museus passaram a investir em materiais que fossem voltados ao ensino, na busca de estabelecer uma boa relação com os professores e as escolas, mas ainda hoje existem muitos museus que não apresentam serviços ou setores voltados para a educação, devido à falta de investimento público (Pereira \& Braga, 2013). Cabe destacar, a importância da participação dos professores de Ensino Fundamental nos espaços museais, pois, a partir de suas experiências individuais e coletivas desenvolvidas nesses locais, podem se inspirar, atualizar os seus conhecimentos, elaborar outros meios mais criativos para o ensino na sala de aula, na constante busca de tornar o processo de ensino mais eficaz e mais atrativo para os alunos (Jacabucci, 2013).

Para Cabral e Guimarães (2020), a relação entre museus e escolas é de suma importância, já que as instituições escolares visitam os museus constantemente, contribuindo para que esses espaços sejam mais do que um complemento das disciplinas escolares. Os museus instigam, inspiram e interrogam os alunos a partir das atividades que desenvolvem, pois não são mais lugares que acumulam artigos, mas atualmente são utilizados para diversão, distração e práticas educativas. Ademais, esses espaços não formais auxiliam fortemente o processo de ensino-aprendizagem, sendo uma alternativa para os professores de ciências trabalharem com os seus alunos, proporcionando um processo de construção do conhecimento mais prazeroso, criativo e significativo (Teixeira et al., 2012).

Além da relevante relação existente entre as escolas e os museus de ciências que auxilia no desenvolvimento de um processo de ensino-aprendizagem mais dinâmico, crítico e eficaz, é importante que esses espaços também alcancem a sociedade como um todo. Segundo Rennie e Johnston (2004), as visitas aos museus possibilitam ao público vivenciar diversas experiências importantes para o desenvolvimento de um indivíduo, trabalhando tanto características sociais, quanto afetivas e culturais. Além disso, a participação do público em geral nos espaços museais, contribui para a aproximação com as ciências e as tecnologias, as quais muitas vezes estão distantes do meio social, favorecendo a melhor compreensão destas, e auxiliando na melhoria da qualidade de vida da população (Souza, 2011).

Segundo Tojal (2015), com o desenvolvimento de movimentos sociais mais fortes relacionados às políticas públicas de inclusão na sociedade brasileira, na década de 1980, um processo de adaptação inclusiva iniciou-se principalmente nas escolas 
públicas. Ainda de acordo com a mesma autora, a busca por inclusão de pessoas com deficiência, seja ela física, sensorial, emocional ou intelectual, também se expandiu para os espaços não formais de educação, como os museus e espaços culturais, os quais passaram a ser visitados por novos frequentadores, mesmo que em baixo número, necessitando modificar tanto sua estruturação física, comunicacional e comportamental para uma inclusão realmente efetiva.

Cabe destacar ainda, os museus de ciências acessíveis, os quais desempenham papel fundamental para a inclusão das pessoas com deficiência nesses espaços de divulgação da ciência. Porém, não são todos os estados brasileiros que apresentam museus adaptados e acessíveis, provocando um baixo número de visitantes desse público. Desse modo, é notório que a maior parte das pesquisas científicas relacionadas aos museus estão mais preocupadas com a aprendizagem e o ensino e não com a inclusão das pessoas com deficiência nesses espaços científicos, sendo necessário um melhor planejamento e adaptação dos museus (Schuindt \& Silveira, 2019).

Outro dado importante observado está relacionado à trabalhos que também investigaram a comunidade escolar lato e stricto sensu. Mesmo se tratando de um museu de ciências, os espaços museais tem como objetivo a sua aproximação com o meio social a qual estes espaços estão inseridos e sendo visitados (Gruzman \& Siqueira, 2007), portanto são ambientes voltados para a comunidade no seu sentido amplo, e não somente restrito a práticas escolares com discentes.

Quanto às ferramentas metodológicas empregadas nas obras investigadas neste estudo, foram identificadas uma ampla diversificação de abordagens, sendo a análise quantitativa $(52,27 \%)$ a metodologia de análise que mais prevaleceu, e questionário $(40,90 \%)$ e entrevista $(34,09 \%)$ como metodologias de coleta mais frequentes (Figura 3). Essa mesma diversidade de instrumentos metodológicos também foi observada em estudo realizado por Schneider, Fujii e Corazza (2017) a partir de análises de publicações sobre ensino de ciências em quatro periódicos. Os autores também encontraram a análise qualitativa como a mais utilizada nos trabalhos analisados.

Figura 3. Abordagens e ferramentas metodológicas de coleta (A) e análise (B) de dados utilizadas nos artigos analisados que abordam ciências e/ou biologia em museus do Brasil.

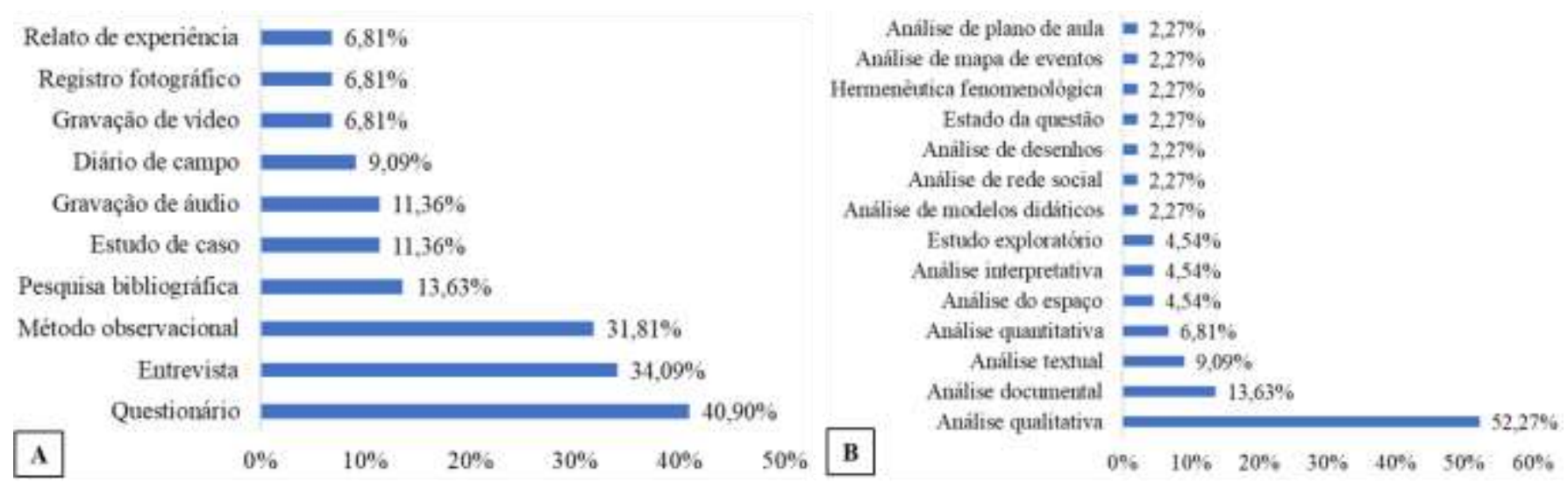

Fonte: Autores (2021).

A maior utilização da análise qualitativa nas pesquisas pode ser justificada pelo seu poder de investigação do sujeito avaliado, conseguindo identificar variáveis, fenômenos e indagações mais próximas possíveis à realidade do investigado e proporcionar ao pesquisador a sua aproximação com esta realidade, para que assim possa compreendê-la e interpretar isto em suas análises (Zanette, 2017).

Duas importantes e comuns ferramentas de levantamento de dados também foram destaque nesta pesquisa, os questionários e entrevistas. Estas são técnicas de coletas de dados primários com foco descritivo na interlocução do público investigado, na qual o questionário permite trabalhar facilmente com um maior número de participantes e abordar de forma mais 
rápida e abrangente diferentes questionamentos; já a entrevista necessita da conversação do entrevistador com o entrevistado, possibilitando adquirir informações mais reais, fidedignas e com maior qualidade de perceber atitudes, reações e condutas (Prodanov \& Freitas, 2013; Oliveira et al., 2016).

Analisando o contexto da educação não formal em museus, é possível destacar a importância de ambas as metodologias de coletas citadas no parágrafo anterior, uma vez que mixadas à análise qualitativa permitem investigar de forma interativa e detalhada os sujeitos da pesquisa, possibilitando o diagnóstico e a orientação de questões educativas de forma geral praticadas nos espaços museais. Isto reflete nos dados encontrados no presente estudo, pois utilizam destas metodologias de coleta e análise para garantir a qualidade nas informações obtidas.

\section{Considerações Finais}

Com base nos dados obtidos a partir do estado da arte, identifica-se diante do cenário geral que poucos são os trabalhos desenvolvidos abordando ciências/biologia em museus no Brasil. Ainda assim, os trabalhos existentes demonstram diversificação quanto aos espaços museais e as ferramentas metodológicas para a obtenção dos dados e público-alvo.

Estes dados, validam informações importantes sobre o perfil destes trabalhos e expõe predominâncias mais que significativas de dados que refletem na relevância dos museus como espaços não formais que articulam, através de suas atividades, diferentes áreas do conhecimento e contemplam diferentes setores da sociedade, com grande potencial para atingir um público amplo e diverso.

Os resultados encontrados reforçam a necessidade de museus como locais de educação para a cidadania, desde a proposição de atividades científicas por funcionários, para professores, alunos, comunidade e como espaço inclusivo para pessoas com deficiência. Igualmente, enfatiza-se a necessidade da ampliação de informações e dos diferentes museus existentes, para que a popularização da ciência continue em evidência e que cada vez mais a sociedade participe de forma ativa nas atividades desenvolvidas pelos museus de ciências/biologia.

Além disso, sugere-se o desenvolvimento de mais trabalhos que relacione os museus e o ensino de Ciências/Biologia especialmente aqueles cujos dados demostram que foram pouco explorados (p. ex. temáticas de Geodiversidade, Astronomia, Microbiologia; público-alvo envolvendo pessoas com deficiência, alunos do ensino superior, comunidade escolar latu sensu; em estados e/ou regiões que apresentam nenhum ou poucos trabalhos; e ecomuseus). Além disso, sugere-se o desenvolvimento de mais estudos como este, no intuito de identificar um panorama mais amplo do que está sendo investigado e trabalhado junto aos espaços museais e seu público frequentador, propondo assim, alternativas e mecanismos mais práticos e que os aproximem mais da sociedade em geral.

\section{Referências}

Bizerra, A. F., Cizauskas, J. B. V., Inglez, G. C. \& De Franco, M. T. (2012). Conversas de aprendizagem em museus de ciências: como os deficientes visuais interpretam os materiais educativos do museu de microbiologia? Revista Educação Especial, 25(42), 57-73.

Cabral, E. D. C. \& Guimarães, V. F. (2020). O Público Potencial Escolar do Museu da Vida: um estudo exploratório em escolas da zona norte da cidade do Rio de Janeiro. Ciência \& Educação, 26, 1-16.

Cancela, C. D. M. \& Carvalho, C. (2017). A divulgação da ciência e o Museu de Astronomia e Ciências Afins. Interacções, 13(44), 43-64.

Coelho, T. B., Lauro, A. T. D., Simões, I. M. de S., Mourão, M. I. A., Mazzoni, M. A. \& Chiavegatto, B. (2019). Museus de História Natural como Ferramentas de Divulgação Científica. Analecta - Centro Universitário Academia, 5(5), 1-16.

Córdova, F. P. \& Silveira, D. T. (2009). A pesquisa Científica. In: T. E. Gerhardt \& D. T. Silveira (Orgs.). Métodos de Pesquisa. Editora da UFRGS.

Costa, R. M. J. Oficina interativa baseada na Educação Ambiental crítica no Museu Espaço Ciência Viva no Rio de Janeiro. 145 f. Dissertação (Mestrado em Biociência e Saúde) - Instituto Oswaldo Cruz, Rio de Janeiro.

Falaschi, R. L., Capellari, R. S. \& Oliveira, S. S. (2011). Museus de ciência: do reconhecimento e conservação da biodiversidade à divulgação científica. Revista Simbio-Logias, 4(6), 12-23. 
Fantin, M., Santos, J. D. A. \& Martins, K. J. (2019). Black Mirror e o espetáculo revisitado: um estado da arte e algumas reflexões. Revista Diálogo Educacional, $19(62), 1147-1173$.

Ferreira, N. S. de A. (2002). As pesquisas denominadas "Estado da Arte". Educação \& Sociedade, 23(79), 257-272.

Gil, A. C. (2008). Pesquisa Social: métodos e técnicas. Atlas.

Gomes, I. \& Cazelli, S. (2016). Formação de Mediadores em Museus de Ciência: Saberes e Práticas. Revista Ensaio: Pesquisa em Educação e Ciências, 18(1), 23-46.

Gruzman, C. \& Siqueira, V. H. F. de. (2007). O papel educacional do Museu de Ciências: desafios e transformações conceituais. Revista Electrónica de Enseñanza de las Ciencias, 6(2), 402-423.

Jacobucci, D. F. C., Nogueira-Ferreira, F. H. \& Santana, F. R. (2013). Representações de educação não formal e utilização do espaço museal por professoras do Ensino Fundamental. Ensino Em Re-Vista, 20(1), 125-132.

Lazzarin, L. F. (2017). Pesquisa em educação. Santa Maria: Núcleo de Tecnologia Educacional.

Leite, P. P. (2016). Ecomuseus e Museologia Social. Informal Museology Studies, 4, 1-98.

Marandino, M., Campos, N. F., Leporo, N., Caffagni, C. W. A., Maia, R. \& Oliveira, A. D. (2012). A percepção de biodiversidade em visitantes de museus: um estudo no Brasil e na Dinamarca antes da visita. Tempo Brasileiro, 188(188), 97-112.

Meyer, G. C. \& Meyer, G. C. (2014). Educação ambiental em museus de ciência: diálogos, práticas e concepções. Revista Brasileira de Educação Ambiental, $9(1), 70-86$

Minayo, M. C. de S. (2010). Pesquisa social: Teoria, método e criatividade. Vozes.

Museu de Astronomia e Ciências Afins. (2021). Sobre o museu. http://www.mast.br/museu/sobre/

Oliveira, J. C. P., Oliveira, A. L., Morais, F. de A. M., Silva, G. M. \& Silva, C. N. M. (2016). O questionário, o formulário e a entrevista como instrumentos de coleta de dados: vantagens e desvantagens do seu uso na pesquisa de campo em ciências humanas. Anais do Congresso Nacional de Educação, Natal, RN, 3.

Ovigli, D. F. B. (2015). Panorama das pesquisas brasileiras sobre educação em museus de ciências. Revista brasileira de estudos pedagógicos, 96(244), 577595.

Ovigli, D. F. B. (2011). Prática de Ensino de Ciências: o museu como espaço formativo. Revista Ensaio: Pesquisa em Educação e Ciências, 13(3), 133-149.

Pereira, J. S. \& Braga, J. L. M. (2013). Museu e experiências docentes. Ensino Em Re-Vista, 20(1), 83-94.

Prodanov, C. C. \& De Freitas, E. C. (2013). Metodologia do Trabalho Científico: Métodos e Técnicas da Pesquisa e do Trabalho Acadêmico. Novo Hamburgo: Feevale.

Pscheidt, C. \& Lorenzetti, L. (2020). Contribuições de um curso de formação continuada para a promoção da alfabetização científica de docentes no Museu da Terra e da Vida. Alexandria: Revista de Educação em Ciência e Tecnologia, 13(1), 155-179.

Pugliese, A. \& Marandino, M. (2015). Os museus de ciências como componente curricular dos cursos de Licenciatura: uma análise sociológica. Anais do Encontro Nacional de Pesquisa em Educação em Ciências, Águas de Lindóia, SP, 10.

Queiroz, R. M., Teixeira, H. B., Veloso, A. dos S., Terán, A. F. \& Queiroz, A. G. (2011). A Caracterização dos espaços não formais de educação científica para o ensino de ciências. Revista Amazônica de Ensino de Ciências, 4(7), 12-23.

Rennie, L. J. \& Johnston, D. J. (2004). The Nature of Learning and Its Implications for Research on Learning from Museums. Science Education, 88(S1), S4S16.

Romanowski, J. P. \& Ens, R. T. (2006). As pesquisas denominadas do tipo "Estado da arte" em educação. Revista Diálogo Educacional, 6(19), 37-50.

Rossi, A. V. (2013). Museu de ciências universitário: sobre espaços de divulgação, educação e produção científica. Ensino Em Re-Vista, 20(1), $209-218$.

Scalfi, G. A. de M., Iszlaji, C. \& Marandino, M. (2020). A formação de professores na perspectiva CTSA por meio de atividades nos museus de ciências. Indagatio Didactica, 12(4),73-90.

Schneider, E. M., Fujii, R. A. X. \& Corazza, M. J. (2017). Pesquisas quali-quantitativas: contribuições para a pesquisa em ensino de ciências. Revista Pesquisa Qualitativa, 5(9), 569-584.

Schuindt, C. C. \& Silveira, C. (2019). Museus de Ciências Acessíveis no Brasil: mapeamento das áreas de conhecimento. Anais do Encontro Nacional de Pesquisa em Educação em Ciências, Natal, RN, 12.

Silva, M. C. B. D. \& Leite, R. C. M. (2020). Formação de professores em museus de ciências: construindo o estado da questão. Atos de Pesquisa em Educação, 15(3), 695-717.

Souza, D. M. V. (2011). Ciência para todos? A divulgação científica em museus. Ciência da Informação, 40(2), 256-265.

Steola, A. C. D. S. \& Kasseboehmer, A. C. (2018). O Espaço da Química nos Centros e Museus de Ciências Brasileiros. Química Nova, 41(9), 1072-1082. 
Research, Society and Development, v. 10, n. 6, e53510616026, 2021

(CC BY 4.0) | ISSN 2525-3409 | DOI: http://dx.doi.org/10.33448/rsd-v10i6.16026

Teixeira, H. B., Queiroz, R. M. D., Ghedin, E., Almeida, D. P. D. \& Terán, A. F. (2012). A inteligência naturalista e a educação em espaços não formais um novo caminho para uma educação científica. Revista Amazônica de Ensino de Ciências, 5(9), 55-66.

Tojal, A. (2015). Política de acessibilidade comunicacional em museus: para quê e para quem. Revista Museologia \& Interdisciplinaridade, 4(7), 190-202.

Valente, M. E. A. (2014). Interseções Necessárias: história, museologia e museus de ciências e tecnologia. Museologia \& Interdisciplinaridade, 3(5), 37-53.

Zanette, M. S. (2017). Pesquisa qualitativa no contexto da Educação no Brasil. Educar em Revista, 33(65), 149-166. 